\title{
Research on High-Tech Virtual Enterprise Integrated Information Management Methods and Systems
}

\author{
Changyuan Gao and Zidan Shan \\ School of Economics and Management, Harbin University of Science and Technology, \\ Harbin 150040, Heilongjiang, P.R. China Gaocy2002@126.com shanzidandana@163.com
}

\begin{abstract}
High-tech Virtual Enterprise (HTVE) is a complicated open tremendous system. It should deal with collaborative manufacture between independent entities with different functions in cooperated enterprises and effective integration of existing information system, business process and commerce rules. This paper combines the characteristics of separated topology organization structure in HTVE to establish its information management model and operational mechanism applying the Distributed Artificial Intelligence approach. Then the Integrated Information Management Systems for Virtual Business in High-tech Enterprise under internet environment is constructed from the aspects of system arrangement, network module and function design. Therefore, the reconfigurable, reusable, scalable and dynamic mutual networkbased cooperative commerce chains are realized.
\end{abstract}

Keywords: High-tech virtual enterprise, Information management mechanism, Integrated information management system, Distributed artificial intelligence, Work flow, Web services

\section{INTRODUCTION}

At present, many countries are exploring and attempting novel growing patterns of high-tech enterprises [1]. Analyzing from the development status and strategic guidance, establishing virtual enterprise should be an effective way to make up deficiency and bring up persistent core competitive ability [2]. High-tech Virtual Enterprise (HTVE) takes the core ability or the superiority ability of high-tech enterprises as support, uses the information technology to obtain the functions that the organization's own resources are not provided with. In addition, it realizes function mutation to meet the dynamic requirements through resource reorganization, function combination, parallel cooperation, and non-property right cooperates with selected correlative enterprises. The new characteristics of HTVE make it difficult to research with traditional management theories and methodologies.

HTVE operation manner requires effective support of integrated information management methods and system platforms. HTVE is a complicated open tremendous system. It should base on entire targets of alliances to deal with distributed and parallel collaborative manufacture among independent entities, as well as effective integration of existing information system, business process and commerce rules. However, existing enterprises have adopted themselves two kinds of integrated

Please use the following format when citing this chapter:

Gao, C., Shan, 7., 2007, in IFIP International Federation for Information Processing. Volume 254, Research and Practical Issues of Enterprise Information Systems II Volume 1, eds. L. Xu. Tjoa A., Chaudhry S. (Boston: Springer). pp. 695-700. 
information management styles: information transmission mode and information centre mode. Neither of these two ways is able to solve the questions of information coordination and information security in HTVE. Distributed Artificial Intelligence (DAI) origins from the late 1970s [3], which is capable of overcoming limitations of resource, space-time distribution and functions existing in single intelligence system, with the advantages of parallel, distribution, openness and fault-tolerance, etc. DAI could meet various demands for processing information in HTVE very well.

Hence, how to reform the integrated information management mode which can guarantee the independence of partners and realize resource sharing in HTVE, and how to comprehensively apply existing information systems of the enterprises and HTVE information management platform, have become key problems restraining HTVE from being successful in management.

\section{HTVE ORGANIZATION AND BEHAVIOR MODEL}

In the distributed system, the organized units have intelligence and autonomy, and are equal in cooperation with each other. The principal and subordinate (sup-sub) between the units of traditional organizations have not been applied. The topological structure solves physically or logically interconnected relationship among units. HTVE is formed by numerous units, divided into core unit organization and non-core unit organization, such as hegemonic enterprises and partnership enterprises. There into, Hegemonic enterprises include original enterprises (VOT) and intimate enterprises (VIT) i.e. VIT $=\left\{V I T_{1}, V I T_{2}, \cdots, V I T_{m}\right\}$. VOT and VIT set up the integrative Management Centre in HTVE $\left(V I M C=\left\{V O T, V I T_{i}\right\} \quad(i=1,2, \cdots, m)\right)$ together, which is responsible for the management, coordination, control, lead and get in touch to the outside of the whole HTVE. In addition, VOT and VIT take on the conductors (VCR) of the virtual project team (VTM) respectively. Different VCR chooses unit (VU) in VTM, the project is decomposed to several sub tasks $V A T=\left\{V A T_{1}, V A T_{2}, V A T_{3}, \cdots, V A T_{m}, V A T_{m+1}\right\}$, which distribute to

$V T M=\left\{V T M_{1}, V T M_{2}, \cdots, V T M_{m}, V T M_{m+1}\right\}$, over here,

$V T M_{i}=\left\{V C R_{j}, V U_{j}\right\}(j=1,2, \cdots, m+1)$, even $V C R=V O T U V I T$,

$V U=V U_{1} \cup V U_{2} \cup \cdots \cup V U_{m} \cup V U_{m+1}$

$=\left\{V U_{1}{ }^{1}, V U_{2}{ }^{1}, \cdots, V U_{k}{ }^{\prime}\right\} \cup\left\{V U_{k+1}{ }^{2}, V U_{k+2}{ }^{2}, \cdots, V U_{k+1}{ }^{2}\right\} \cdots \cup\left\{V U_{r+1}{ }^{m+1}, \cdots, V U_{r+s}{ }^{m+1}\right\}$

After finishing sub tasks, VTM would disaggregate, so VCR turns to regression state.

It is especially important to establish interior and exterior HTVE information behavior model for constructing the integrated information management system (IMS) and exploring the distributional artificial intelligence technology (such as Figure 1). Correspondingly, HTVE information behavior model includes conceptual model, process model and coordinated model. (1)The conceptual models are composed of VCR model and VU model. (2)The process model is the information modeling among VTMs in HTVE. (3)The coordinated models deal with synthesis management of VTMs to form coordinated and harmonious decision-making process. 


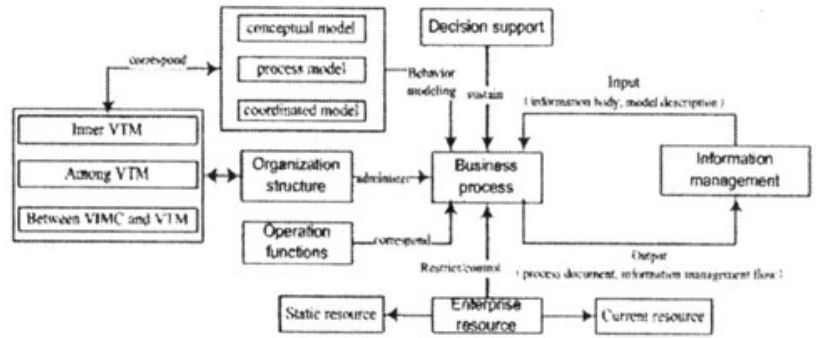

Figure 1. Relations of HTVE Information Behavior Model

\section{INTEGRATED IMS OF HTVE}

The design standards of the system structure meet the characteristic of DAI and have very high flexibility [3]. Construction of design should proceed from three different angles corresponding to HTVE information behavior mode, first, enterprise's information management system inside VTM, second, enterprise's information management system between VTM, and third, enterprise's information management system between VIMC and VTM.

\subsection{Entire Architecture and Network Modules of HTVE Integrated IMS}

The paper bases on information technologies and relevant standards of Internet, Web Service, distributed Work Flow etc. It proposes four-layer integrationinformation-management-system-framework (Figure 2), including operation control layer, decision support layer, user problem dealing layer, and technology support layer. The Four layers structure of the system is suitable for the information management system of VOT and VIT, within VTM (between VCR and many VUs) and between VTM.

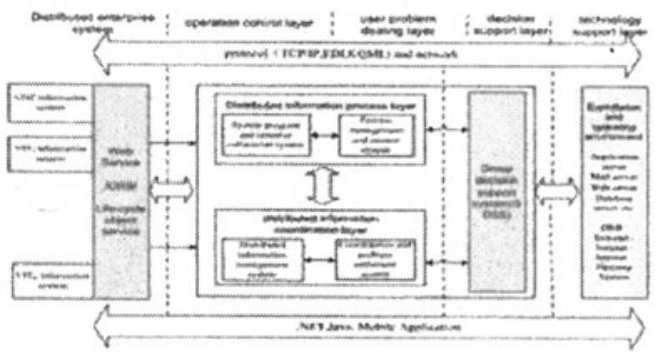

Figure 2. Four Layers Structure of HTVE Integrated Information Management System

In order to support the operation of HTVE distributed information management, each member enterprise acts as one node in network, possessing inside information system and coordination system and DSS between enterprises. Divide each node into five pieces of module (Figure 3). Internal module (IM) represents an independent 
autonomy member, including the information management system and production control system of VIT, VOT and VU, administering intact information structure, all internal decision-making processes and enterprise activity inside member's enterprises. IM forms the information process layer. Distributed Information Management Module (DIMM) symbolizes the module that manages information among different organizations and with VOT and VIT, and within VTM, and between VTM. CM and DIMM form information coordination layer, and is mainly controlled by VIMC. Decision Module (DM) deals with non-structural problems among the enterprises of the members, DM forms the decision support layer. Communication Infrastructure (CI) supports mutual agreements between HTVE nodes. In correspondence with inside and outside organizations, it divides into Internal Communication Protocol (ICP) and External Communication Protocol (ECP). CI forms the technology support layer.

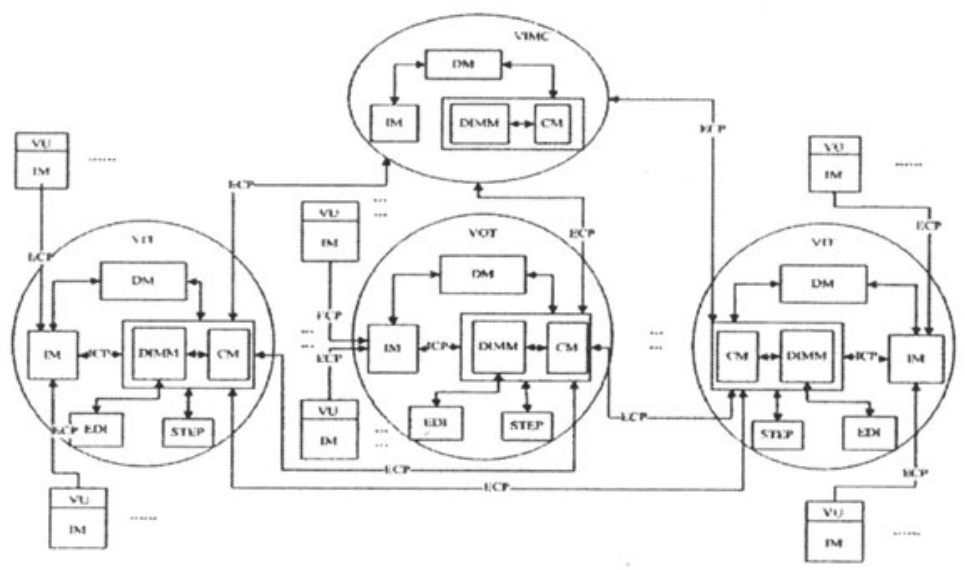

Figure 3. Information Network Module Structure in HTVE

Operation control layer is composed of information process layer and information coordination layer. The information process layer is mainly about the analysis and design of IM. IM is divided two parts. The former is to carry out reasonable plans and distributions to organizations, resources, information database of VOT, VIT, and VU. The later completes the tasks and orders from former, controls the implementations of tasks in real-time, while feedback to program and resource collocation system takes prompt action. Information coordination layer concerns itself mainly of analyses and design of DIMM and CM. DIMM. It exchanges information process through ICP with IM, and its partial outputs for CM, DM and other DIMM to use. With the support of workflow management system, CM transmits workflow data, which deal with work processing engine from distributed process management information of IM to integrated process management system of $\mathrm{CM}$, finally finishes supporting processrunning status by enterprise task lists shown. The agreement management system guarantees the normal operation of HTVE integrated information management system, and supervises and controls its course. Decision support system depicts the 
Research on High-Tech Virtual Enterprise Integrated Information Management Methods and

Systems

design of DM. Spacial and systematical distribution determines that the decision support system is GDSS.Technology support layer is formed by supporting milieu, system service, exploitation environment and the tools [4]. Based on the differences between cooperating relationship and the consanguineous grades of member enterprises, it offers mail service and ORB [5] which is systematic encapsulating tool.

\subsection{Function Design in HTVE Integrated IMS}

According to the characteristics of HTVE whole architecture and network modules constructed by the integrated information management system in hegemony enterprises and partner enterprises, the paper describes functions of three different entities as VU, VCR and VIMC (Figure 4). HTVE public information centre as workspace needs to truss up the large mainstream quantities of application software platforms, and also support various basic platforms and application server for VU, VCR and VIMC.

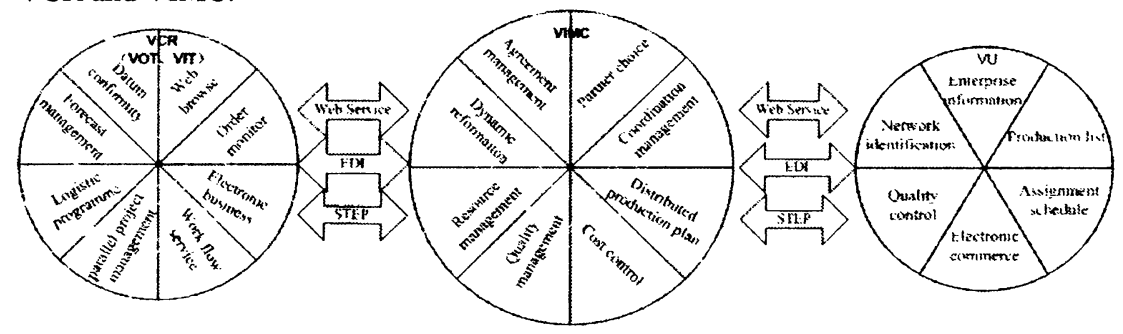

Figure 4. Function Design Frame of VU, VCR and VIMC

\section{CONCLUSIONS}

HTVE Integrated information management system applies DAI method, expanded by high-tech enterprise existing systems, which guarantees the independence of member enterprises and keeps the relevance of the entrepreneurial alliances. The core technology of member enterprises is mostly left in IM, the users inside the enterprise can only visit its information, and other enterprises can only visit the information in DIMM and CM. Accordingly, the reconfigurable, reusable, scalable and dynamic mutual network-based cooperative commerce chains are realized. However, on account of dynamic and complex organization in HTVE, we need to explore and research more, i.e. negotiation mechanism of and members' credit in HTVE etc. 


\section{ACKNOWLEDGEMENTS}

This research was supported by two projects: (1) Project 70373058 supported by NSFC (2) Project 06D070 supported by Heilongjiang Social Science Foundation.

\section{REFERENCES}

1. C. Henry, W. Lau, and E.T. Wong. Partner Selection and Information Infrastructure of a Virtual Enterprise Network, Int J. Integr Manuf.. Volume 14, Number 2, pp.186-193, (2001).

2. R.E. Giachetti, A Framework to Review the Information Integration of the Enterprise, Int J. Product Res.. Volume 42, Number 6, pp.1147-1166, (2004).

3. K.H. Choi, D.S. Kim, and Y.H. Doh, Multi-agent-based Task Assignment System for Virtual Enterprises, Robot Comput Integr Manuf.. Volume 2, Number 3, pp.1-6, (2007).

4. B.G. Bernsttein and W. Ruh, Enterprise Integration: the Essential Guide to Integration Solutions (Addison-Wesley: Boston, MA, 2005)

5. V. Ermolayev and N. Keberle, Towards a Framework for Agent-enabled Semantic Web Service Composition, Int J. Web Serv Res.. Volume 1, Number 3, pp.63-87, (2004). 